\title{
An 11.4-Mb Interstitial Deletion in a Fetus with No Apparent Phenotypic Alterations
}

\author{
Paolo Guanciali-Franchi ${ }^{a, c}$ Claudio Celentano ${ }^{b}$ Melissa Alfonsi ${ }^{a, c}$ \\ Chiara Palka ${ }^{d}$ Giulietta Di Pasqua ${ }^{a, c}$ Barbara Matarrelli ${ }^{b}$ \\ Giandomenico Palka ${ }^{a, c}$ \\ Departments of a Medical Genetics and ${ }^{\mathrm{b}}$ Obstetrics and Gynecology, SS Annunziata Hospital, and Departments of \\ 'Medical Genetics and d Pediatrics, University G. D'Annunzio Chieti-Pescara, Chieti, Italy
}

\section{Key Words}

Array $\mathrm{CGH} \cdot$ Chromosome $8 \cdot$ Interstitial deletion

\begin{abstract}
A prenatal case of a de novo interstitial deletion distal to $8 q 24$ was reported. Ultrasound examination and postmortem evaluation demonstrated no apparent phenotypic alterations. Array CGH showed an 11.4-Mb loss in chromosome 8 ranging from $8 q 24.13$ to $8 q 24.23$. This case partially overlaps the 2 cases previously described in the literature.
\end{abstract}

๑) 2016 S. Karger AG, Basel

Interstitial deletions or microdeletions at $8 \mathrm{q} 24$ could be recognizable in syndromes such as Langer-Giedion syndrome (LGS), mild LGS-like phenotype, or multiple cartilaginous exostoses of long bones with facial hypertrichosis and epilepsy [Verheij et al., 2009]. Only 3 cases with a mosaic interstitial deletion in $8 \mathrm{q}$, but proximal to 8q24.13, have been reported in the literature [Verheij et al., 2009]. Reports on deletions distal to 8q24.13 are unusual, and few cases are reported in the literature [Fennell

\section{KARGER}

๑) 2016 S. Karger AG, Basel

E-Mail karger@karger.com

www.karger.com/msy et al., 1998; Batanian et al., 2001; Verheij et al., 2009]. The clinical manifestations associated with these aberrations range from a mild phenotype with no obvious stigmata of LGS to multiple congenital anomalies, albeit with a larger deleted region. Furthermore, in one such case, a deletion of the c-MYC gene was reported with an apparent normal phenotype [Batanian et al., 2001].

We report a de novo interstitial deletion distal to $8 \mathrm{q} 24.13$ detected in a fetus with a normal ultrasound morphology and postmortem phenotype.

\section{Case Report}

A 35-year-old woman (gravida 2, para 1) was referred to our unit at 16 weeks' gestation to perform an amniocentesis because of advanced maternal age. The family history and the previous pregnancy were uneventful. After an ultrasound evaluation and extensive counseling, a triple screen test for Down syndrome was carried out. Albeit it was negative both for trisomy 18 and 21 (AFP 2.49 MoM; hCG 0.50 MoM, and uE3 1.60 MoM), the patient opted for an amniocentesis. Cytogenetic analysis of amniocytes using GTGbanding showed a 46,XY,del(8)(q24.1q24.2) karyotype in all 20 examined cells (Fig. 1A). Both parents had a normal chromosome complement. 
Ultrasound examination at 12 (Fig. 2A), 16, and 19 weeks' gestation (Fig. 2B) revealed no major fetal anomalies. Family history was unremarkable for pregnancy loss.

A further chromosome study performed on fetal blood obtained by cordocentesis at 19 weeks' gestation showed the same deletion in chromosome 8. FISH analysis using a c-MYC Break Apart probe (Cytocell, Cambridge, UK) showed a hybridizing signal of c-MYC in one chromosome 8 (Fig. 1B). Genomic DNA was extracted from fetal blood using the QIAamp DNA Blood Mini Kit (QIAGEN).

Array CGH (aCGH) was performed using CytoChip Oligo ISCA $4 \times 44 \mathrm{~K}$, analyzed with BlueFuse Multi v4.1 software (BlueGnome, Cambridge, UK), and a 11.4-Mb loss in chromosome 8 ranging from $8 \mathrm{q} 24.13$ to $8 \mathrm{q} 24.23$ at position $125,093,582-$ $136,519,662$ was detected. There are 24 OMIM genes in the deleted area (Fig. 1C).

After extensive genetic counseling, the couple opted to terminate pregnancy due to the high risk of LGS. A further aCGH with DNA obtained from fibroblasts confirmed the diagnosis of del(8) (q24.13q24.23)

The postmortem evaluation demonstrated a normal male fetus consistent to 19 weeks of gestation. The phenotype was normal with no signs of LGS, not even mild symptoms (Fig. 2C).

\section{Discussion}

Several patients with interstitial deletions in the long arm of chromosome 8 proximal to 8q24.13 were reported. Clinical phenotypes of such patients have a high degree of variability depending on the size and position of the de-

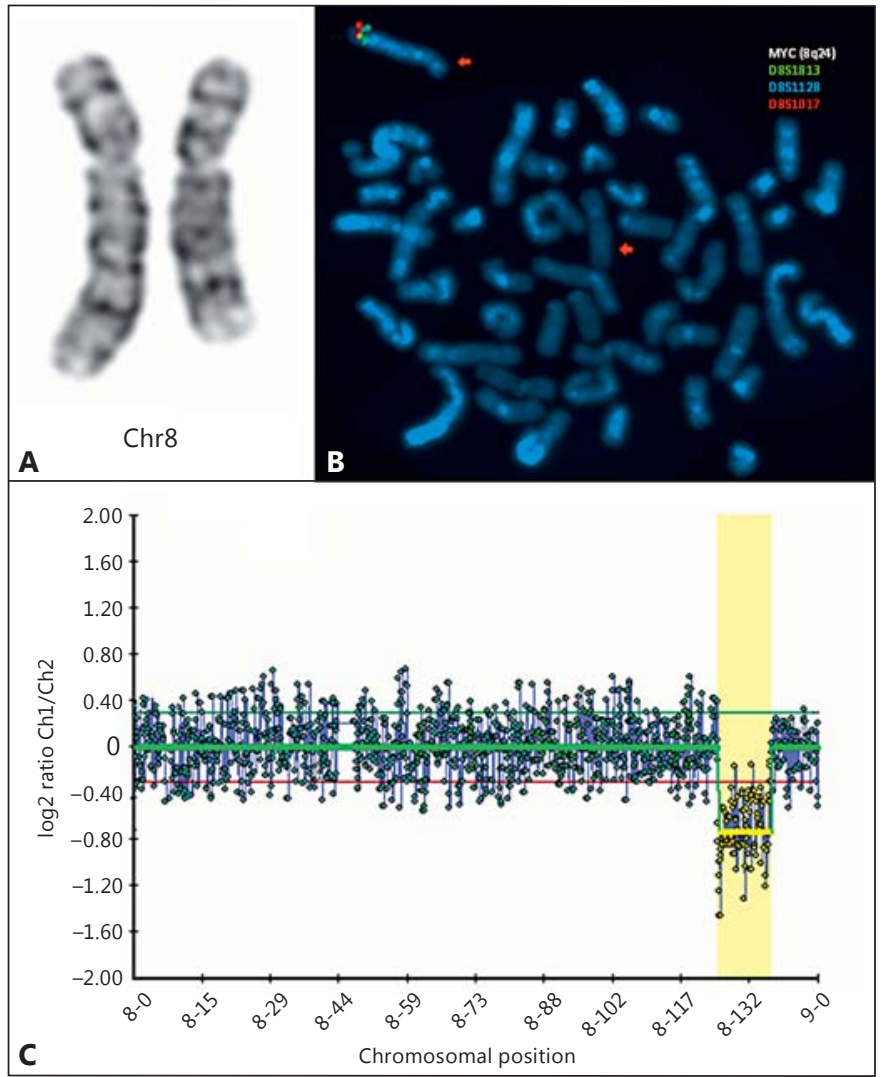

Fig. 1. A G-banded chromosomes 8. B FISH analysis of the proband with the 8q24 microdeletion; only 1 chromosome 8 shows hybridization signals. C aCGH analysis.
Fig. 2. Ultrasound picture at $12(\mathbf{A})$ and at 19 weeks' gestation (B). Phenotypic appearance of the fetus $(\mathbf{C})$.
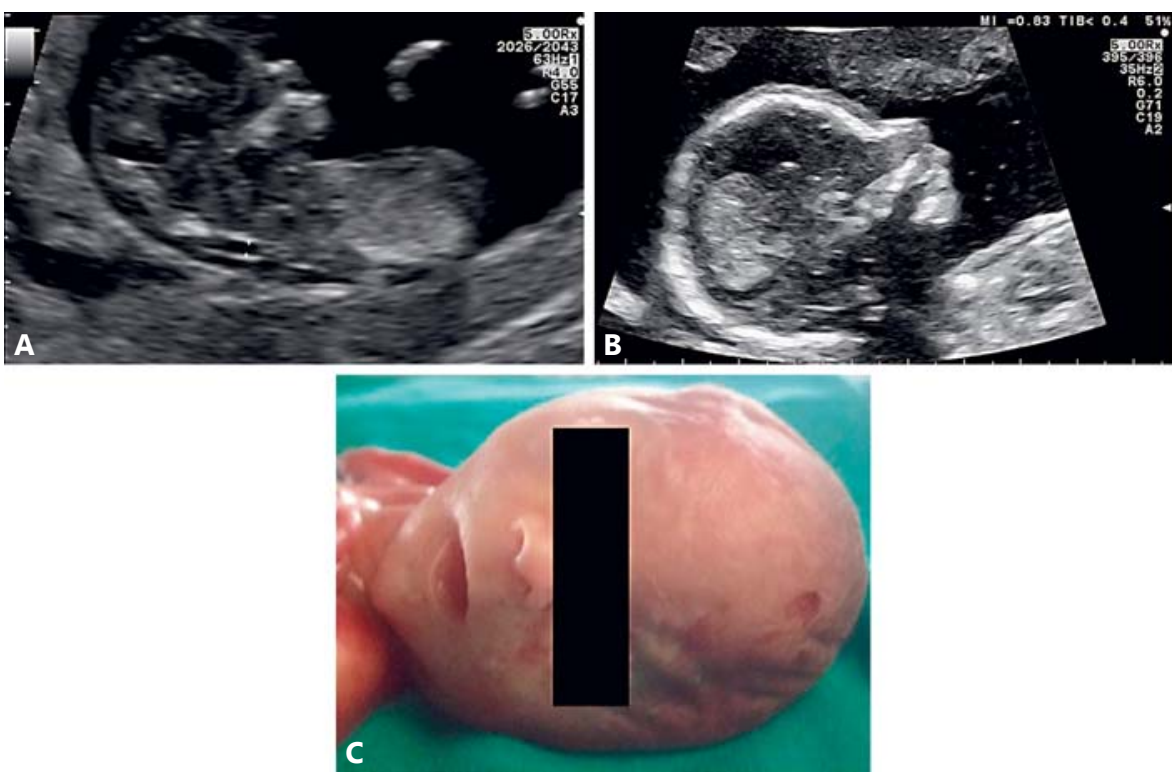

Mol Syndromol 2017;8:42-44 DOI: $10.1159 / 000452967$ 
leted region. In addition, few reports are available on deletions distal to 8q24.13. In the 2 cases reported by Verheij et al. [2009], 1 patient had a del(8)(q24.21q24.3) with multiple congenital malformations, mental delay, and seizures. The other patient carried a $\operatorname{del}(8)$ (q23.2q24.23) with dysmorphic features reminiscent of LGS and seizures. The cases described above are the only cases studied with aCGH [Verheij et al., 2009]. Batanian et al. [2001] reported a fetus carrying a $\operatorname{del}(8)(\mathrm{q} 24.13 \mathrm{q} 24.22)$ aborted at 26 weeks' gestation without apparent malformations investigated with standard cytogenetic and FISH analysis. The same deletion was found in the phenotypically normal mother. She had a history of miscarriage and fetal loss. Fennel et al. [1989] reported 2 de novo cases with a mild phenotype carrying a deletion involving the distal part of 8q24.1, however, only using classical cytogenetic studies.

The present case partially overlaps the 2 cases reported by Verheij et al. [2009] from 8q24.21 to 8q24.23 in a de novo interstitial deletion. Usually, chromosomal deletions involving euchromatic areas are associated with abnormal phenotypes, although some specific chromosomal regions are much more associated with normal clinical aspects [Verheij et al., 2009]. There are 24 OMIM genes in the 11.4-Mb deleted region in our patient, distal to the TRSP1 and EXT1 genes. Eight OMIM diseases are mapped in the same region: Renal cell carcinoma (RCC; OMIM 144700), spastic paraplegia 8 (SPG8; OMIM 603563), Burkitt lymphoma (BL; 113970), seizures (BFNS2; 121201), ciliary diskinesia (CILD19; 614935), autoimmune thyroid disease 3 (AITD3; 608175), thyroid dyshormonogenesis 3 (THD3; 274700), and Charcot-MarieTooth disease type 4D (CMT4D; 601455) (OMIM; http:// www.omim.org). Clinical manifestations of all these diseases are not prenatally detectable. Deletions of the cMYC gene are reported in hematologic disorders as well as in normal individuals [Batanian et al., 2001]. Moreover, c-MYC expression is required for a normal facial morphogenesis [Uslu et al., 2014], albeit the ultrasound scan and the postmortem evaluation were unremarkable for any dysmorphisms. Although a first trimester screening was not performed, and thus an evaluation of the predictive values, e.g., pregnancy-associated plasma proteinA and free beta-hCG [Padula et al., 2016] (either as absolute risk or associated to maternal age) as well as an uterine doppler velocimetry evaluation [Cignini et al., 2016] was not possible, she had a normal second trimester biochemical test. At 16 and 19 weeks' gestation, fetal growth was consistent with gestational age, and there were no anomalies sonographically detectable as a genetic sonogram. The deletion of KCNQ3 and spastic paraplegia endorse the high risk of seizures in the present case [Verheij et al., 2009].

\section{Conclusions}

The present case confirmed the necessity of a rapid second level genetic evaluation in fetuses presenting de novo and recurrent small cytogenetic lesions. FISH analysis and aCGH are widely available at the present time to confirm the genes involved. Due to genetic library, a multidisciplinary counseling is suggested to improve parents' awareness and pregnancy management.

\section{Statement of Ethics}

The research was ethically conducted in accordance with the World Medical Association Declaration of Helsinki. The parents have given their informed written consent.

\section{Disclosure Statement}

The authors certify that they are neither affiliated with nor involved in any way with any organization having financial interests in the subject matter discussed in this paper.

\section{References}

Batanian JR, Morris K, Ma E, Huang Y, McComb J: Familial deletion of (8)(q24.13q24.22) associated with a normal phenotype. Clin Genet 60:371-373 (2001)

Cignini P, Maggio Savasta L, Gulino FA, Vitale SG, Mangiafico L, et al: Predictive value of pregnancy-associated plasma protein-A (PAPP-A) and free beta-hCG on fetal growth restriction: results of a prospective study. Arch Gynecol Obstet 293:1227-1233 (2016).
Fennell SJ, Benson JW, Kindley AD, Schwarz MJ, Czepulkowski B: Partial deletion 8q without Langer-Giedion syndrome: a recognisable syndrome. J Med Genet 26:167-171 (1989).

Padula F, Laganà AS, Vitale SG, D'Emidio L, Coco $\mathrm{C}$, et al: The introduction of the absolute risk for the detection of fetal aneuploidies in the first-trimester screening. J Matern Fetal Neonatal Med 21:1-5 (2016).
Uslu VV, Petretich M, Ruf S, Langenfeld K, Fonseca NA, et al: Long-range enhancers regulating $M y c$ expression are required for normal facial morphogenesis. Nat Genet 46:753-758 (2014).

Verheij JB, de Munnik SA, Dijkhuizen T, de Leeuw N, Olde Weghuis D, et al: An 8.35 Mb overlapping interstitial deletion of 8q24 in two patients with coloboma, congenital heart defect, limb abnormalities, psychomotor retardation and convulsions. Eur J Med Genet 52:353-357 (2009).
44

Mol Syndromol 2017;8:42-44 DOI: $10.1159 / 000452967$
Guanciali-Franchi/Celentano/Alfonsi/

Palka/Di Pasqua/Matarrelli/Palka 